SCIENTIFIC LETTER

\title{
Intracoronary brachytherapy for the treatment of complex in-stent restenosis
}

\author{
J M Cotton, K Rance, A Patil, M R Thomas
}

Heart 2005;91:231-232. doi: 10.1136/hrt.2003.028886

$\mathrm{T}$ he development of an effective strategy for management of in-stent restenosis (ISRS) following "bare metal" stenting has proved difficult. Standard percutaneous therapy for ISRS leads to re-restenosis rates above $50 \%$ and consequently represents a poor treatment option. ${ }^{1}$ At present the "gold standard" treatment for ISRS is vascular brachytherapy (VBT), with re-restenosis rates falling to below $20 \%$ in most trials. ${ }^{2}{ }^{3}$ While the use of drug eluting stents (DES) appears to be effective in simple ISRS lesions, the results in complex cases are less impressive. ${ }^{45}$

At present, however, there are no published data on the clinical utility of VBT in complex native coronary lesions and multivessel disease. We have therefore analysed the procedural details and clinical outcome of patients with complex ISRS treated with intracoronary brachytherapy at our institution.

\section{METHODS}

A total of 126 consecutive patients treated at our institution with ISRS from August 2000 to February 2003 were assessed. Cases were deemed complex if:

- there was multi-vessel ISRS or

- ISRS involving a bifurcation, or

- ISRS associated with complete coronary occlusion (no cases of acute/subacute stent thrombosis were included).

Clinical and procedural data were collected prospectively and clinical follow up was by telephone interview at $6,12,18$, and 24 months.

A total of 39 patients were identified with complex ISRS. Sixteen were treated for ISRS effecting two vessels (eight with true birfurcational lesions) and 23 patients were treated for ISRS with a total coronary occlusion. Of the 39, four presented with unstable symptoms ( $>3$ months after their index stent procedure) and four had left ventricular dysfunction with a left ventricular ejection fraction of $<40 \%$. Ten subjects had previously undergone coronary artery bypass surgery, and 10 had diabetes mellitus.

All patients received aspirin $300 \mathrm{mg}$ and clopidogrel $300 \mathrm{mg}$. Procedural anticoagulation was achieved by the administration of 10000 units of heparin. Abciximab was used in $16 \%$ of the occlusion cases and $18 \%$ of the bifurcation cases.

The Novoste $\beta$-rail catheter (Novoste Europe, Belgium) system was used in all cases. A cutting balloon was used where practicable before VBT. When treating bifurcation lesions with two doses of brachytherapy, care was taken to avoid two full doses of $\beta$ radiation at the crux (fig 1 ). The brachytherapy source was initially placed in the parent vessel across the bifurcation (fig 1B); following this the source was passed down the sidebranch and the proximal marker carefully positioned at the crux (fig $\mathrm{IC}$ ) Using this technique the risk of full double dosing to the crux of the bifurcation was minimised.

\section{Definitions}

- ISRS: > 50\% diameter stenosis by quantitative coronary angiography (QCA) within a previously stented vessel

- Bifurcation: ISRS lesions involving a parent vessel and significant side branch $>2 \mathrm{~mm}$ in diameter

- Myocardial infarction (MI): a rise in creatine kinase (CK) to greater than twice the upper limit of normal in our institution

- Major adverse cardiac event (MACE): death, myocardial infarction, emergency coronary artery bypass graft surgery, or target vessel revascularisation (TVR)

$\beta$ Radiation doses were calculated as per the vessel diameter. The $\beta$ source train was constructed with ${ }^{90} \mathrm{Sr} /{ }^{90} \mathrm{Y}$. Quantitative coronary angiography (QCA) was performed offline. All patients received clopidogrel for at least one year.

Continuous variables referred to in this text are expressed as mean (SD) unless otherwise stated.

\section{RESULTS}

Fifty five vessels were treated in 39 patients. Mean age was 61 years (range $38-80$ years). Ten $(25.6 \%)$ were diabetic, 10 $(25.6 \%)$ had undergone previous coronary artery bypass grafting (CABG), and four (10.3\%) were female. Four patients $(10.6 \%)$ had reduced left ventricular function and four $(10.6 \%$, all with multivessel ISRS) had presented with an unstable coronary syndrome.

Twenty three patients underwent VBT for ISRS with coronary occlusion. The mean time to intracoronary brachytherapy from the index procedure was 11.9 (7.1) months.

Procedural success was $96 \%$. Follow up data were available for all patients at a mean of 12.5 months (0-27 months). Mean reference diameter pre-procedure was 2.8 (0.3) $\mathrm{mm}$ and the minimum luminal diameter post-procedure was 2.2 (0.5) mm. In-hospital major adverse cardiac event (MACE) was $4.3 \%$ and follow up MACE $8.7 \%$.

One patient suffered a non-Q myocardial infarction (MI) periprocedurally, with a full recovery. One patient required target vessel (but not target lesion) revascularisation at 11 months. The failure occurred in a saphenous vein graft lesion whereby following deployment of the brachytherapy catheter there was no reflow which failed to respond to further intervention. This occurred without clinical event and there was no enzyme rise.

Abbreviations: $C A B G$, coronary artery bypass grafting; $D E S$, drug eluting stents; ISRS, in-stent restenosis; QCA, quantitative coronary angiography; VBT, vascular brachytherapy 

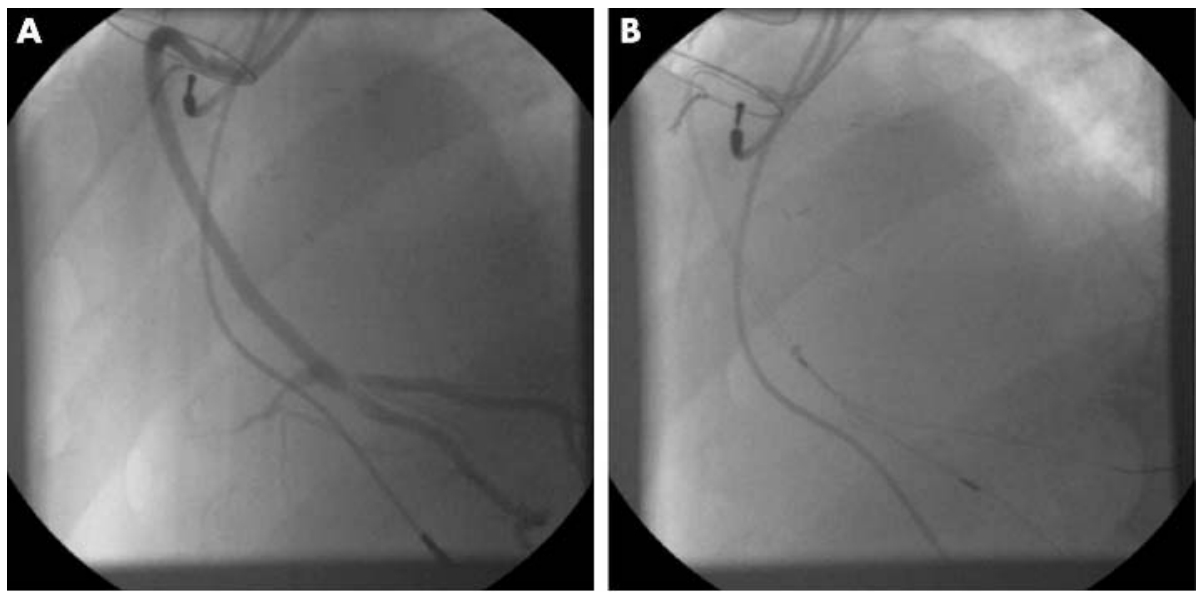

Figure 1 Brachytherapy procedure to ISRS lesion at insertion of saphenous vein graft to the distal right coronary branches. (A) Pre-procedure. (B) Brachytherapy source to parent vessel. (C) Brachytherapy source to branch with careful positioning at crux. (D) Final result.
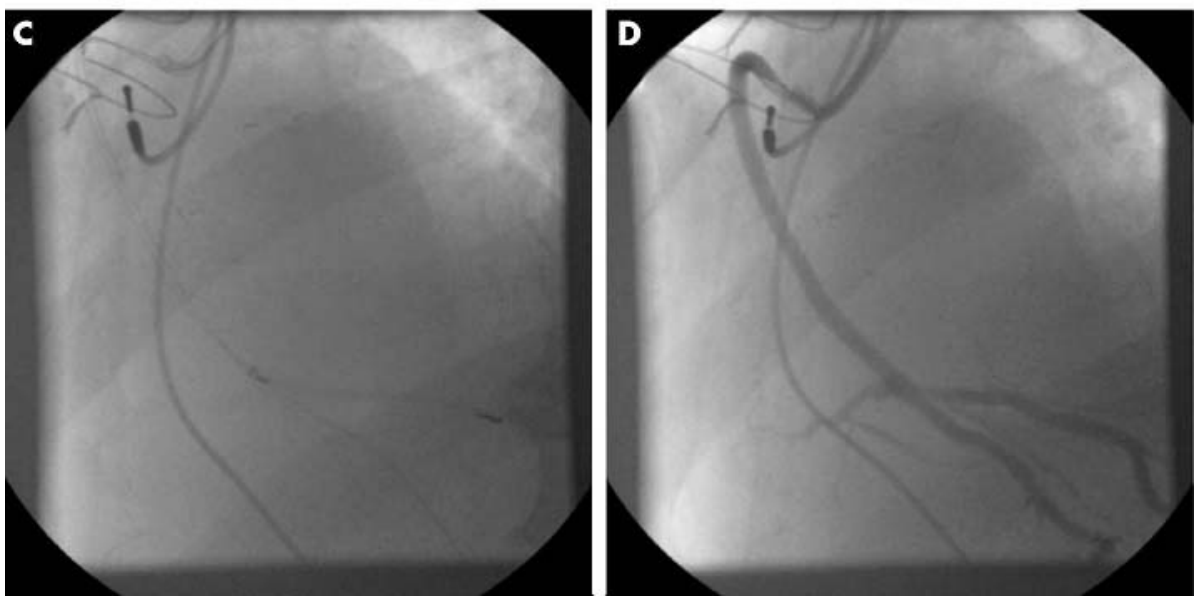

Sixteen patients with multivessel disease were treated (with lesions in 32 vessels): eight patients had true bifurcation lesions, and eight had two vessel ISRS. The left main stem was involved in three cases, one protected and two unprotected. The left anterior descending artery was involved as either the parent or side branch vessel in 10/16 cases.

Procedural success was $100 \%$ with no inpatient MACE.

At mean follow up of 10.8 months (0-24 months), one patient had died and two others required target vessel revascularisation (MACE 19\%). The death occurred suddenly six weeks post-procedure in a 79 year old female patient with unprotected left main stem restenosis, a chronically occluded right coronary artery, and concurrent renal failure.

\section{DISCUSSION}

The short-mid term follow up MACE rates described in this series $(8.7 \%$ for occlusions and $19 \%$ for double vessel and bifurcation lesions) compare favourably with previous brachytherapy trials in more straightforward lesions. At present the use of DES for ISRS is controversial, with differing results depending on lesion complexity. A randomised controlled trial of IVBT versus DES for this indication is awaited.

The principal concern of brachytherapy in multi-vessel ISR is the delivered radiation dose. We have demonstrated that, at least in this early experience, there appear to be no clinical sequelae at a mean follow up of 11.7 months. The relative risks and benefits of the treatment options for complex ISRS need to be considered on a case by case basis. At present we believe that the results of IVBT as evidenced by this series are acceptable and compare favourably with both DES and CABG in this difficult subset of patients.

\section{Authors' affiliations}

J M Cotton, K Rance, A Patil, M R Thomas, Department of Cardiology, King's College Hospital, London, UK

Correspondence to: Dr Martyn R Thomas, Department of Cardiology, Kings College Hospital, Denmark Hill, London. SE5 9RS; mttwins@ aol.com

Accepted 1 March 2004

\section{REFERENCES}

1 Baim DS, Levine MJ, Leon MB, et al. Management of restenosis within the Palmaz-Schatz coronary stent (the U.S. multicenter experience). The U.S. Palmaz-Schatz stent investigators. Am J Cardiol 1993;71:364-6.

2 Waksman R, White RL, Chan RC, et al. Intracoronary gamma-radiation therapy after angioplasty inhibits recurrence in patients with in-stent restenosis. Circulation 2000;101:2165-71.

3 Waksman R, Bhargava B, White L, et al. Intracoronary beta-radiation therapy inhibits recurrence of in-stent restenosis. Circulation 2000;101:1895-8.

4 Sousa JE, Costa MA, Abizaid A, et al. Sirolimus-eluting stent for the treatment of in-stent restenosis: a quantitative coronary angiography and threedimensional intravascular ultrasound study. Circulation 2003;107:24-7.

5 Degertekin M, Regar E, Tanabe K, et al. Sirolimus-eluting stent for treatment of complex in-stent restenosis. The first clinical experience. J Am Coll Cardiol 2003;41:184-9. 